\title{
ORIENTATION OF NEURITE GROWTH BY EXTRACELLULAR ELECTRIC FIELDS ${ }^{1}$
}

\author{
NILESH PATEL AND MU-MING POO ${ }^{2}$ \\ Department of Physiology and Biophysics, University of California, Irvine, California 92717
}

Received September 8, 1981; Revised October 23, 1981; Accepted November 24, 1981

\begin{abstract}
Extracellularly applied sleady electric fields of 0.1 to $10 \mathrm{~V} / \mathrm{cm}$ were found to have marked effects on the neurite growth of single dissociated Xenopus neurons in culture: (1) neurites facing the cathode showed accelerated growth, while the growth of those facing the anode was reduced. Neurites growing relatively perpendicular to the field axis were prompted to curve toward the cathode. (2) More neurites appeared to be initiated from the cathodal side of the cell. (3) The number of neurite-bearing neurons per culture and the average neurite length were increased. These effects are absent in cultures treated with electric fields of similar strength but alternating polarity and cannot be attributed either to a gradient of extracellular diffusible substances or to the flow of culture medium produced by the field. The field effects are reversible: (1) removal of the electric field resulted in the loss of neurite crientation in a few hours and (2) reversal of the polarity of the electric field led to a rapid reversal in the neurite orientation. To determine the cellular loci of these field effects, we treated the neurons with a number of pharmacological agents or altered their ionic environments. Incubation with concanavalin A (Con A) was found to abolish these field effects completely. Since the binding of Con A to the neuronal surface was shown to prevent field-induced accumulation of the Con A receptors toward the cathodal side of these neurons, our finding is consistent with the notion that cathodal accumulation of growth-controlling surface glycoproteins by the field is the underlying mechanism of the field-induced orientation of neurite growth toward the cathode.
\end{abstract}

It is generally believed that gradients of physical and chemical factors in the extracellular environment play a major role in determining the direction of neurite growth (for a review, see Jacobson, 1978). One type of physical factor is the presence of weak extracellular electric fields within the tissue. Several previous studies (Marsh and Beams, 1946; Sisken and Smith, 1975; Jaffe and Poo, 1979) have demonstrated that neurite outgrowths from explanted nervous tissue in culture were directed preferentially toward the cathode of an extracellularly applied electric field. Quantitative analysis (Jaffe and Poo, 1979) indicated that an electric field of 0.7 to $1.4 \mathrm{~V} / \mathrm{cm}$ induced neurites which faced the cathode to grow several times faster than those facing the anode.

There are three major problems confronting this line of research. First, one needs to determine whether the electric field exerts its effect directly on the neuron or indirectly through alterations of physical or chemical

\footnotetext{
' This work was supported by National Institutes of Health Grant NS 14483-02. We thank Dr. S. H. Young for the help in computer analyses and Drs. S. E. Fraser, S. H. Young, and I. Chow for comments on the manuscript.

${ }^{2}$ To whom correspondence should be addressed.
}

factors in the extracellular environment of the neuron. Secondly, one needs to localize the cellular loci of the electric field effects. Finally, one needs to determine whether there is any physiological significance of these field effects for neurite growth in vivo. As a first step in tackling these problems, it is imperative to characterize the electric field effects quantitatively on isolated neurons in a controlled environment, where the electric field near the surface of the neuron can be determined accurately, and the resultant cellular events can be examined directly.

In the present study, we used dissociated Xenopus embryonic neurons for a quantitative characterization of the effects of the electric field on neurite growth. We found that steady electric fields, on the order of $1 \mathrm{~V} / \mathrm{cm}$, produced three types of growth responses in these cells: (1) the neurite growth toward the cathode was accelerated, while growth toward the anode was reduced. Neurites growing relatively perpendicularly to the field axis curved toward the cathode, a response initiated by a "bending" at the growing tip. (2) More neurites appeared to be initiated on the cathodal side of the soma. (3) The number of neurite-bearing neurons per culture and the average neurite length were increased. Control experi- 
ments have been performed to exclude the possibility that these field effects were due to the formation of a gradient of substances in the culture medium or to the medium flow as a result of the field application. In addition, we have investigated possible cellular mechanisms underlying these field effects. We found that the electric field used in these studies caused a "lateral electrophoresis" (Poo, 1981) of the concanavalin A (Con A) receptors on the surface of these neurons toward the cathodal side of the cell. This field-induced accumulation of the Con A receptors is probably responsible for the orientation of neurite growth toward the cathode since the immobilization of the Con A receptors by the binding of Con A to the cells abolished the asymmetric growth response of the neurites to the field. Taken together, our findings support the notion that the observed neurite orientation results from the direct action of the electric field on neuronal membrane, possibly by redistributing surface glycoproteins which are responsible for controlling the neurite growth or the adhesion of the neurite to the substratum. Preliminary results of this study have been reported previously in a brief form (Patel and Poo, 1979). During the course of this work, another report on the field effects on isolated Xenopus neurons also has appeared (Hinkle et al., 1981).

\section{Materials and Methods}

\section{Cell culture and application of the electric field}

Fertilized eggs from mature Xenopus laevis were obtained by conventional procedures. When the eggs developed to stage 17 to 19 (Nieuwkoop and Faber, 1962), the anterior portion of the neural tube was removed and dissociated according to the method of Jones and Elsdale (1963). Single cells from the dissociated neural tube were plated in thin rectangular culture chambers and used for experiments $30 \mathrm{~min}$ after plating. The rectangular culture chamber of defined geometry $(60 \times 10 \times 0.18 \mathrm{~mm})$ was made by sealing (with silicon grease) pieces of No. 1 cover glass to the bottom of a tissue culture dish (Corning) to form the side walls. The culture medium was Steinberg's saline $(58.0 \mathrm{~mm} \mathrm{NaCl}, 0.7 \mathrm{~mm} \mathrm{KCl}, 0.4 \mathrm{~mm}$ $\mathrm{Ca}\left(\mathrm{NO}_{3}\right)_{2}, 0.1 \mathrm{~mm} \mathrm{MgSO}_{4}$, and $4.6 \mathrm{~mm}$ Tris) supplemented with $10 \%$ Leibovitz medium (Gibco) and 5\% fetal bovine serum (Gibco). The $\mathrm{pH}$ of the medium was 7.8 . When the dissociated cells had adhered to the bottom of the chamber (15 min after plating), the roof of the chamber (No. 1 cover glass) was placed on gently and sealed to the side walls with silicon grease. At this stage, most of the dissociated cells were spherical in shape and no neurites were observed. The electric field was applied to the culture chamber via a pair of salt bridges made with culture medium gelled by $2 \%$ agar. The methods of applying the electric field were identical to those reported previously (Poo et al., 1978). All electric field applications were carried out in an incubator maintained at room temperature $\left(21 \pm 1^{\circ} \mathrm{C}\right)$. After removal of the field, the cells were fixed immediately with $1 \%$ glutaraldehyde and preserved in $95 \%$ glycerol.

\section{Analysis of neurite growth}

Line drawings of isolated neurons and their neurite processes were made with the aid of a camera lucida fitted to an inverted phase contrast microscope (Nikon). A composite graph of the neurite growth was obtained by placing the center of the cell body of each neuron at the origin of a polar coordinate graph paper with the direction of the electric field set along the $0^{\circ}$-to- $180^{\circ}$ axis of the graph. With the aid of a digitizer (Houston Instrument) and a microcomputer (Cromenco, Z-2D), three types of indices were computed to characterize the growth of the neurite processes. All of the neurite-bearing cells in the culture were included in our analysis. Since these Xenopus cultures contain a heterogeneous population of different cell types, neurons that fail to send out neurites are indistinguishable from other non-neuronal spherical cells and hence are not assayed in the present analysis.

Average neurite length (ANL). To assay the average length of neurite growth under various experimental conditions, the trajectory of each process as recorded with a camera lucida was traced by a digitizer. The total length of growth (including all of the branches) then was computed by the microcomputer. The average neurite length (in micrometers) was obtained by dividing the total length by the number of neurites traced.

Neurite growth asymmetry (NGA). The asymmetry in the neurite growth that resulted from the exposure to an electric field was assayed by obtaining the average projections of the processes onto the field axis toward the cathode $\left(\bar{X}_{c}\right)$ and toward the anode $\left(\bar{X}_{a}\right)$.

$$
\bar{X}_{c}=\sum_{i=1}^{N} X_{i c} / N
$$

and

$$
\bar{X}_{t a}=\sum_{j=1}^{M} X_{j a} / M
$$

where $X_{i c}$ and $X_{j a}$ are the projections of individual neurites toward the cathode and anode, respectively. $N$ and $M$ are the total numbers of neurites facing the cathode and anode of the field, respectively. For branched neurites, the projections of the individual branches from the branch point to the growing tip were added to the projection of the main neurite. The index for neurite growth asymmetry (NGA) is defined as

$$
\mathrm{NGA}=\frac{\bar{X}_{c}-\bar{X}_{a}}{\bar{X}_{c}+\bar{X}_{a}}
$$

Note that the index has a value of 0 if the average projected growth toward the anode and cathode are equal; it has a value of 1 or -1 if the average projected growth toward the anode or cathode is equal to 0 . NGA values above 0.10 were considered to represent significant asymmetry (see Tables I and II), since above this asymmetry index value, asymmetric neurite growth could be easily discerned by visual examination of the composite line tracings, such as that shown in Figure 1.

Neurite initiation site asymmetry (NISA). To characterize the distribution of the points of neurite outgrowth from the cell bodies of the neurons, an asymmetry index for neurite initiation sites (NISA) was defined by taking the average of the cosine of outgrowth angles from the cell bodies (Jaffe, 1958):

$$
\mathrm{NISA}=\frac{\sum_{i=1}^{N} \cos \theta_{i}}{N}
$$


where $N$ is the total number of outgrowths and $\cos \theta_{i}$ is the cosine of the outgrowth angle for neurite $i$. Notc if the neurite initiation sites were all confined to the cathodal or anodal pole of the cell bodies, NISA would be close to 1 or -1 , indicating complete polarization toward the cathode or anode. If they were distributed evenly, the index would have a value of 0 . For the same reason as that for neurite growth asymmetry, in the present study, NISA values above 0.10 were considered to indicate significant asymmetry (see Tables I and II).

\section{Results}

Neurite growth in the presence of extracellular electric fields. Neurite growth from dissociated Xenopus neurons in the absence of an electric field showed no preferential orientation either with respect to the neurite initiation site on the cell bodies or the direction of neurite growth. Composite tracings of the cells showed a roughly symmetric pattern of growth (Fig. $1 A$ ). Cultures treated with an electric field of $2.5 \mathrm{~V} / \mathrm{cm}$ for $6 \mathrm{hr}$ showed marked asymmetry in the neurite growth (Fig. $1 B$ ). Longer neurites, many of which curved toward the cathode, were seen on the cathodal side.

A qualitative analysis of growth asymmetry was carried out by constructing two types of computer-assisted plots from the neurite growth data. We plotted (1) the radial distance of the growth cone from the center of the cell body with respect to the angular position of the growth cone (Fig. 2, $A$ and $B$ ) and (2) the frequency of neurite initiation from the cell body at $10^{\circ}$ angular sectors around the perimeter of cell bodies (Fig. 2, $C$ and $D$ ). Relative to controls, this analysis clearly suggests that there were significantly more growth cones and longer neurites toward the cathode and a higher frequency of neurite initiation sites on the cathodal side of the cell body. It is interesting to note that there also appears to be a relatively high number of growth cones as well as initiation sites located at the anodal side of the cell. This is probably due to two reasons. First, as shown in a later section, neurites facing directly toward the anode did not "bend" and curve toward the cathode; rather their growth was greatly reduced, leaving neurites still oriented toward the anode (Figs. 3 and 4). Secondly, a large fraction $(\sim 37 \%)$ of the neurons in our culture were bipolar, and a stimulation of outgrowth at the cathode pole of the cell also might initiate outgrowth on the opposite side due to some inherent property of neurite initiation in the bipolar cell.

To take into account the curvature and the total amount of neurite growth that occurred in the culture, quantitative analyses of the results were carried out by measuring the average neurite length (ANL), neurite growth asymmetry (NGA), and neurite initiation site asymmetry (NISA) as defined under "Materials and Methods." Table I shows the result obtained for a series of cultures exposed to field strengths from 1 to $10 \mathrm{~V} / \mathrm{cm}$ for $6 \mathrm{hr}$. It is clear that the electric fields markedly promoted neurite growth toward the cathode. For the highest field employed $(10 \mathrm{~V} / \mathrm{cm})$, the average projected neuritc length toward the cathode side was nearly doubled, while it was reduced on the anodal side as compared to the control growth. More neurites also appeared to be initiated from the cathodal side of the cell body. More- over, the average length of neurites (irrespective of their orientation) also increased in the presence of the field. This indicates that the field not only orients the direction of neurite growth but also promotes the net neurite extension. In the presence of the field, the average number of neurite-bearing cells per culture also increased from 30 neurons per culture in the control to 61 neurons per culture after exposure to a $10 \mathrm{~V} / \mathrm{cm}$ field for $6 \mathrm{hr}$.

The higher average projected length toward the cathode $\left(\bar{X}_{c}\right)$ did not result only from the curving of neurites toward the cathode. Compared to the control, the total length of the neurites directly growing toward the cathode (without curving response) was increased but was reduced for the neurites directly growing toward the anode. Although this was suggested by the fact that the average neurite length in the field-treated cultures was higher than that of the control, an analysis of neurite length was carried out for neurites directly facing the cathode and anode (outgrowth angle within $45^{\circ}$ sector toward either pole). The total trajectory covered by the neurites after $6 \mathrm{hr}$ in a field of $2.5 \mathrm{~V} / \mathrm{cm}$ was examined

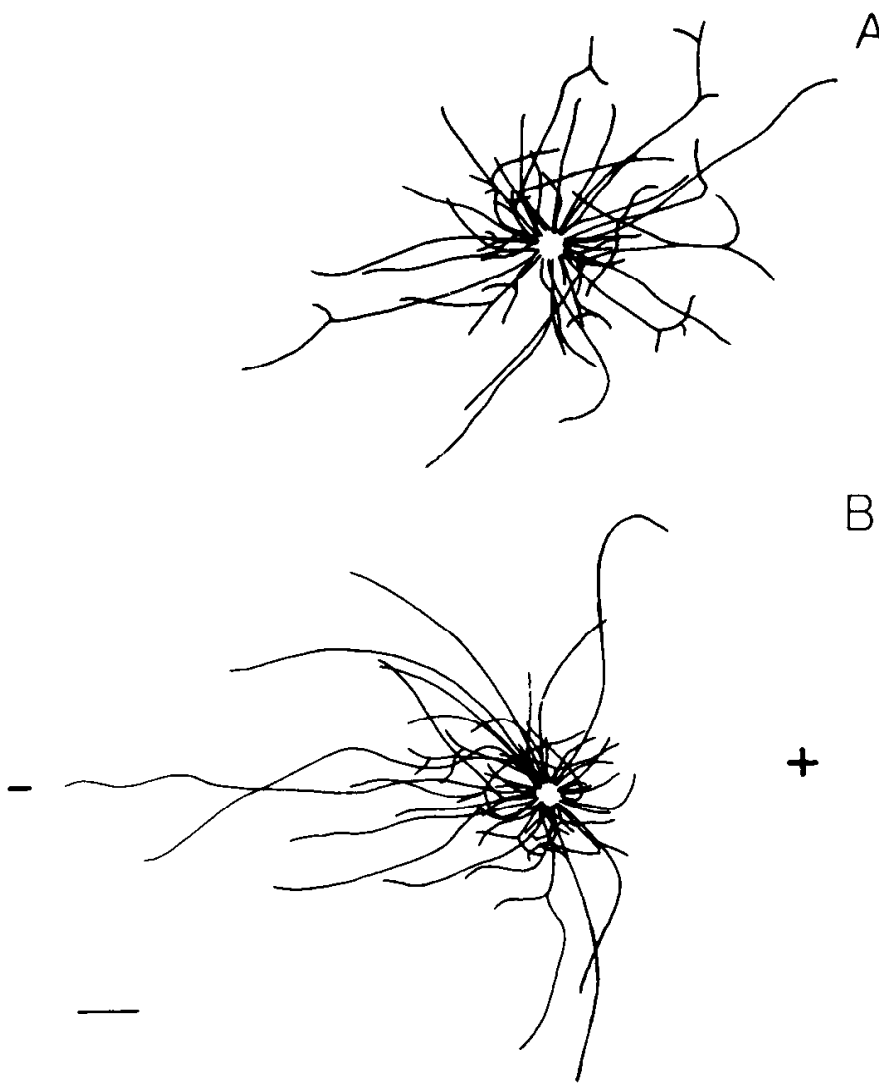

Figure 1. Composite line drawings of neurite growth in cultures of Xenopus neural tube cells. The neurite outgrowth from each isolated neuron was traced with the aid of a camera lucida. The composite drawing was made by superimposing the center of the cell bodies of individual neurons. $A$, Composite drawing of 30 randomly sampled neurons in a $6-\mathrm{hr}$ control culture (not exposed to the electric fields). Indices for neurite growth asymmetry and neurite initiation sites asymmetry were 0.04 and 0.05 , respectively. $B$, Composite drawing of 30 randomly sampled neurites in a culture that was exposed to a field of $2.5 \mathrm{~V} /$ cm for $6 \mathrm{hr}$. Neurite growth asymmetry was 0.34 and neurite initiation site asymmetry was 0.14 . - indicates cathode; + indicates anode. $B a r, 50 \mu \mathrm{m}$. 

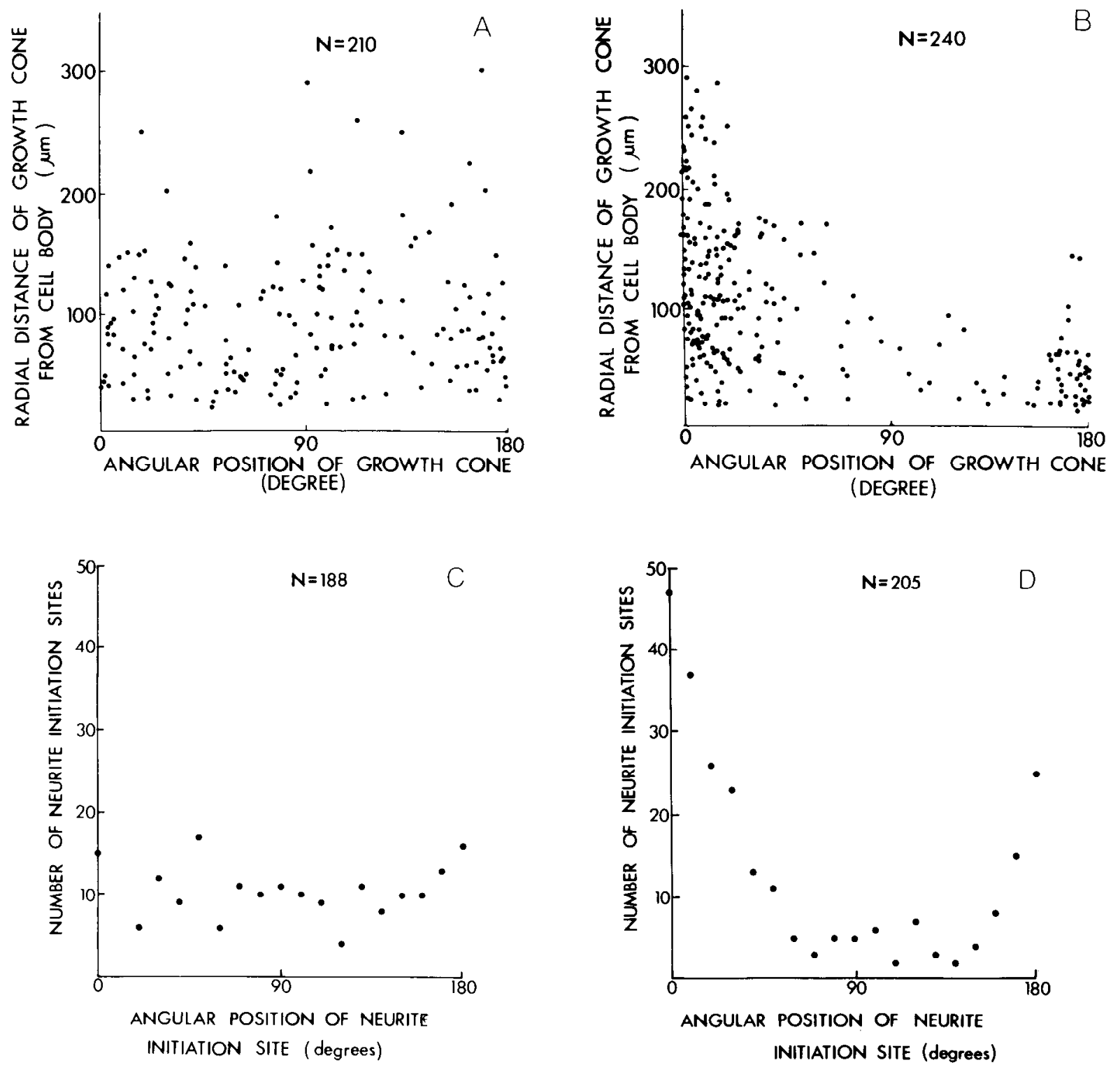

Figure 2. The angular distribution of the radial distances of neurite growth cones from the center of the cell bodies ( $A$ and $B$ ) and the number of neurite initiation sites within every $10^{\circ}$ sector on the surface of the cell bodies $(C$ and $D)$. The angular positions of the cathode and anode are $0^{\circ}$ and $180^{\circ}$, respectively. $A$ and $C$, Data pooled from five 24-hr controls (not exposed to the field). $B$ and $D$, Data pooled from three 24 -hr cultures after $24 \mathrm{hr}$ exposure to a field of $1 \mathrm{~V} / \mathrm{cm}$. Because of the branching of the neurite, the number of measurements $(N)$ of growth cones exceeded that of the initiation sites. Note the orientation of growth cones and neurite initialion siles toward the cathode in the field-treated cultures. The slightly higher frequency toward the anode is discussed in the text.

and compared to the control cultures. Neurites growing directly toward the cathode had an average length of $122.2 \pm 13.9 \mu \mathrm{m}$ (mean $\pm \mathrm{SEM}, N=76$, four cultures), while those growing directly toward the anode had an average length of $71.1 \pm 8.3 \mu \mathrm{m}$ (mean $\pm \mathrm{SEM}, N=54$, four cultures). Thus, as compared to the average neurite length in 6 -hr control cultures $(92.1 \pm 7.2 \mu \mathrm{m})$, there was a $32 \%$ increase and a $23 \%$ reduction in the neurite growth rates for cathode-facing and anode-facing neurites, respectively.

Electric fields of similar amplitude but alternating polarity produced no asymmetry in neurite growth or in the neurite initiation site, also shown in Table I. The average length of neurite growth in the alternating field of a frequency of $0.1 \mathrm{~Hz}$ was reduced slightly.
The orienting effects of the electric field did not persist after the removal of the field. Neurons treated with a field of 5 or $10 \mathrm{~V} / \mathrm{cm}$ for $2 \mathrm{hr}$ showed a neurite growth asymmetry of 0.16 and 0.18 , respectively (numbers of neurites examined, 40 and 58), immediately following removal of the field. However, no neurite growth asymmetry was detected $4 \mathrm{hr}$ later (NGA indices, -0.07 and 0.09 , respectively) in these cultures, suggesting the asymmetry created by the 2 -hr field does not persist. Nevertheless, cultures that had been exposed to the field for 2 $\mathrm{hr}$ generally had a higher average neurite growth and a higher total number of neurites per culture as compared to the control cultures, indicating that both the growth activity and the survival rate of these neurons were increased by the 2-hr treatment with the field. 
TABLE I

Response of embryonic Xenopus neurons to different steady electric fields, alternating electric fields, ${ }^{a}$ and fields with an imposed medium flow $^{b}$

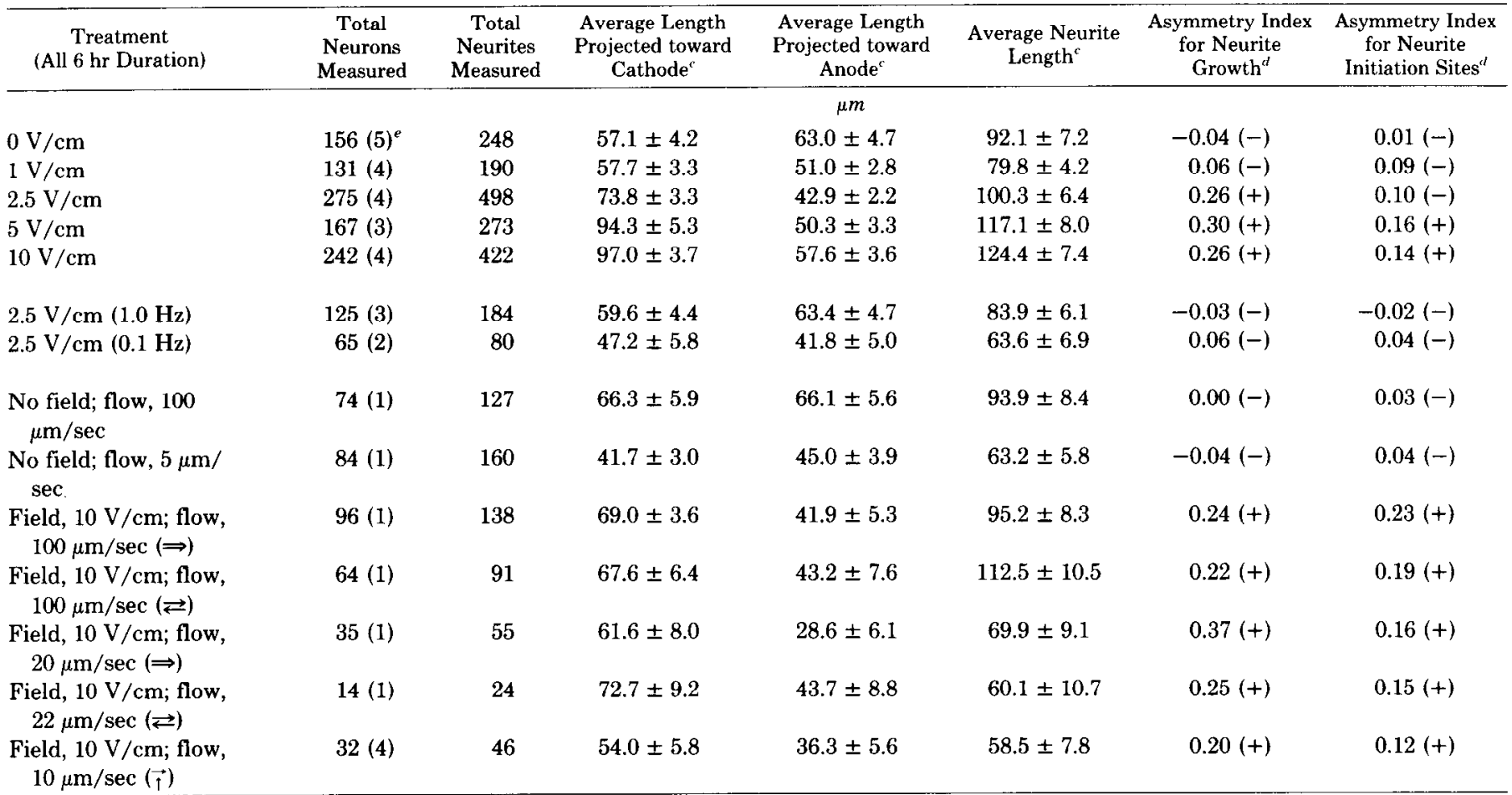

${ }^{a}$ An alternating field was imposed by reversing the polarity of the uniform field with a frequency of $1.0 \mathrm{or} 0.1 \mathrm{~Hz}$.

${ }^{b}$ Medium flow was imposed through the chamber in the same direction $(\Rightarrow)$, the opposite direction $(\rightleftarrows)$, or the perpendicular direction $(\vec{\uparrow})$ with respect to the direction of the electric field.

"Values represent the mean \pm SEM.

${ }^{\prime}$ Asymmetry indices were defined under "Materials and Methods." Indices $\leq 0.10$ were chosen to represent insignificant asymmetry ( - ), and indices $>0.10$ indicate significant asymmetry $(+)$.

- Number of cultures examined.

Orienting effect on the growing neurite. The previous section described the neurite growth observed after $6 \mathrm{hr}$ exposure to an electric field that was imposed before any neurite had appeared. To examine directly the neurite growth responses, fields were applied after substantial neurite growth had occurred, and the growth activity of the neurites was monitored by time-lapse microphotography. As illustrated by Figures 3 and 4, four characteristic responses were observed consistently after the onset of a field of $5 \mathrm{~V} / \mathrm{cm}$ : (1) if the initial orientation of the neurite was relatively perpendicular to the field axis, the field caused the neurite to curve and grow toward the cathode. The curving of the neurite apparently was initiated at the growth cone region, since clear bending of the growing tip was observed before any curvature of the neurite could be seen. A typical bending response is depicted in Figures 3 and 4. In the absence of the field, cells rarely exhibited abrupt and extensive bending of neurites. For bipolar neurons, neurite growth, with respect to the neurite length as well as the branching pattern, was roughly symmetric about the cell body (Fig. 5). (2) Neurites that were oriented initially within about a $30^{\circ}$ sector with respect to the anode had their growth inhibited by the field. Prolonged treatments of the field caused retraction of the neurites, which frequently resulted in the disappearance of the neurite on the anodal side of the cell body (Fig. 4). (3) Neurites facing directly toward the cathode grew continuously at a higher rate along the field axis (Figs. 3 and 4). (4) Reversal of the polarity of the field resulted, within $1 \mathrm{hr}$, in a reorientation of neurite direction and increased growth rate toward the new cathode (Figs. 3 and 4). This relatively rapid reversal of the growth response of the neurites suggests that the field did not cause any permanent alteration in the growth capability of the neurites.

Time-lapse microphotography revealed that, in many cases, there was migration of the cell body either toward the anode or the cathode of the applied electric field. The average projected cell migration along the field axis in a field of $5 \mathrm{~V} / \mathrm{cm}$, as determined from composite tracing of the position of the cell body with respect to fixed markers on the substratum, was about $4.8 \mu \mathrm{m} / \mathrm{hr}$ toward the cathode (average of 27 measurements). This net migration of the cell bodies toward the cathode excludes the possibility that the observed asymmetry in neurite length on the cathodal and anodal sides of the cell was due to the sliding of cell bodies along the neurites or the stretching of the neurites by the cell bodies. Both of these two mechanisms would only decrease the observed asymmetry in neurite growth. Interestingly, in many cases, neurite extension toward the cathode continues as the cell body migrates, maintaining relatively constant neurite lengths (compare Fig. $4, B$ and $C, H$ and $I$ ).

Effects of constant perfusion of culture medium. The 

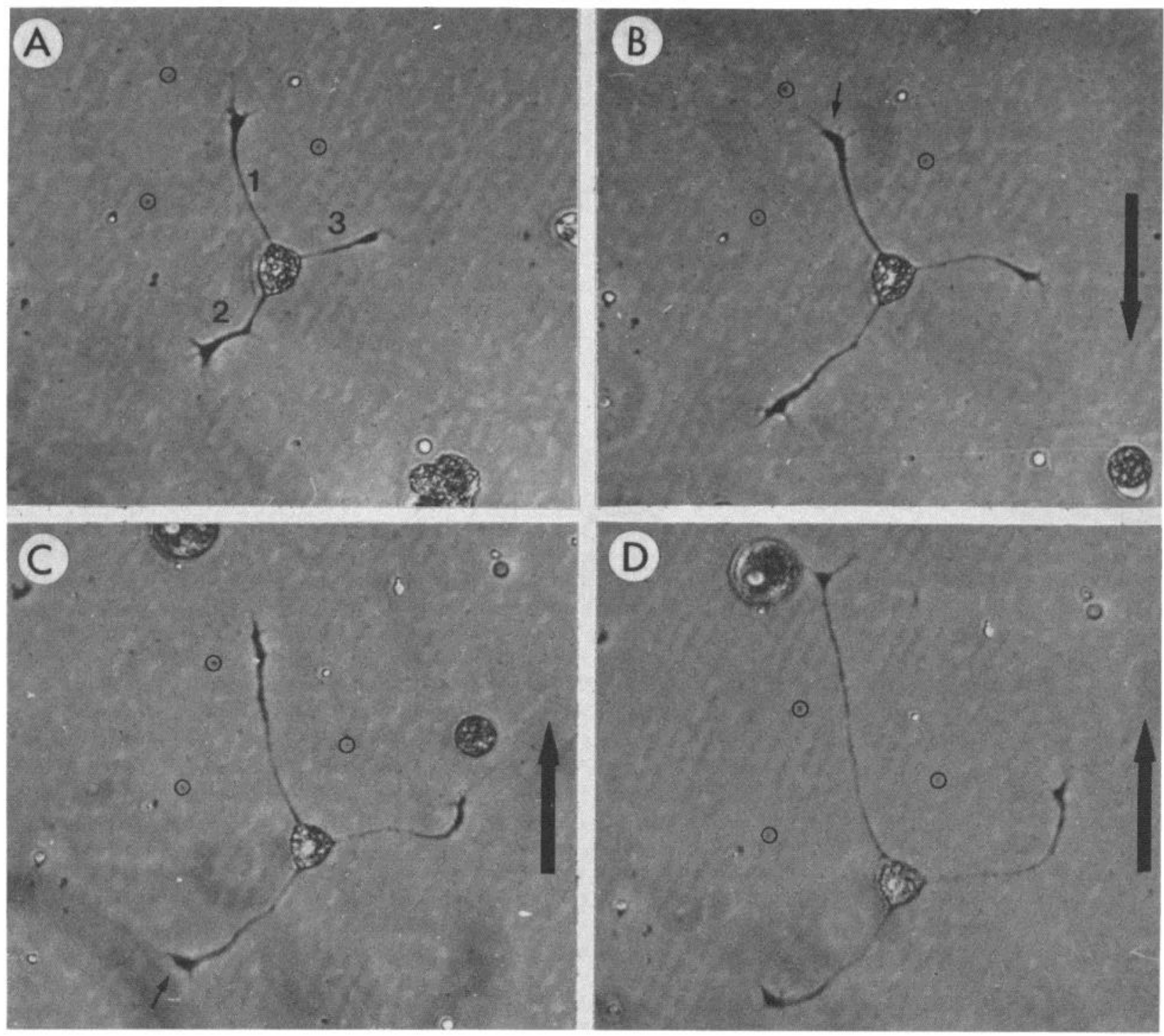

E

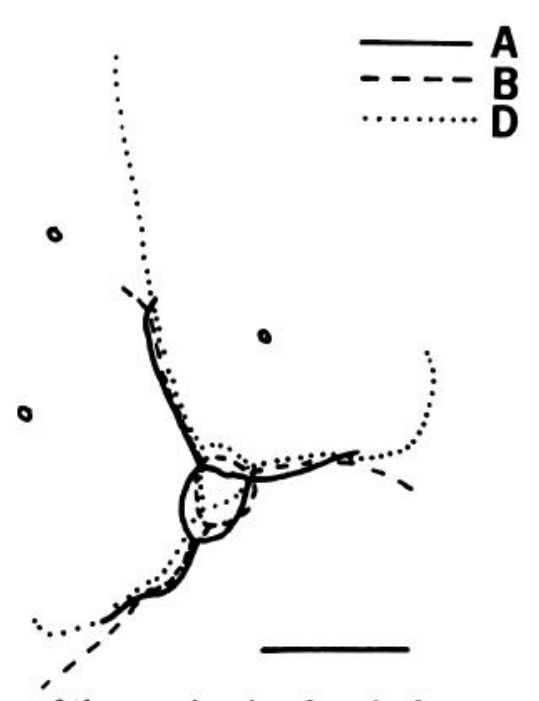

Figure 3. Asymmetric neurite growth and bending of the neurite tip of a tripolar neuron (labeled 1,2 , and 3 ) in the presence of an electric field. $A$, Neurite growth at the start of the experiment $6 \mathrm{hr}$ after plating of the cell. $B, 1 \mathrm{hr}$ after the exposure to a field of $5 \mathrm{~V} / \mathrm{cm}$. The growth of neurite 1 was inhibited (small arrow) and that of neurite 2 was promoted. Neurite 3 curved toward the cathode. The polarity of the electric field was reversed immediately after the photograph in $B$ was taken. $C, 1$ hr after field reversal, neurite 1 resumed growth, while neurite 2 ( small arrow) was inhibited. Neurite 3 bent toward the new cathode. $D$, $2 \mathrm{hr}$ after field reversal, substantial growth was observed in neurite 1, bending appeared at the tip of neurite 2 , and neurite 3 grew toward the cathode. $E$, Composite tracing of the neuron shown in $A, B$, and $D$. Thick arrows present the direction of the field. Small circles refer to fixed markers in the culture substratum. Note that the migration of the cell body was insignificant compared to the asymmetric growth of the neurites. Bar, $50 \mu \mathrm{m}$. 

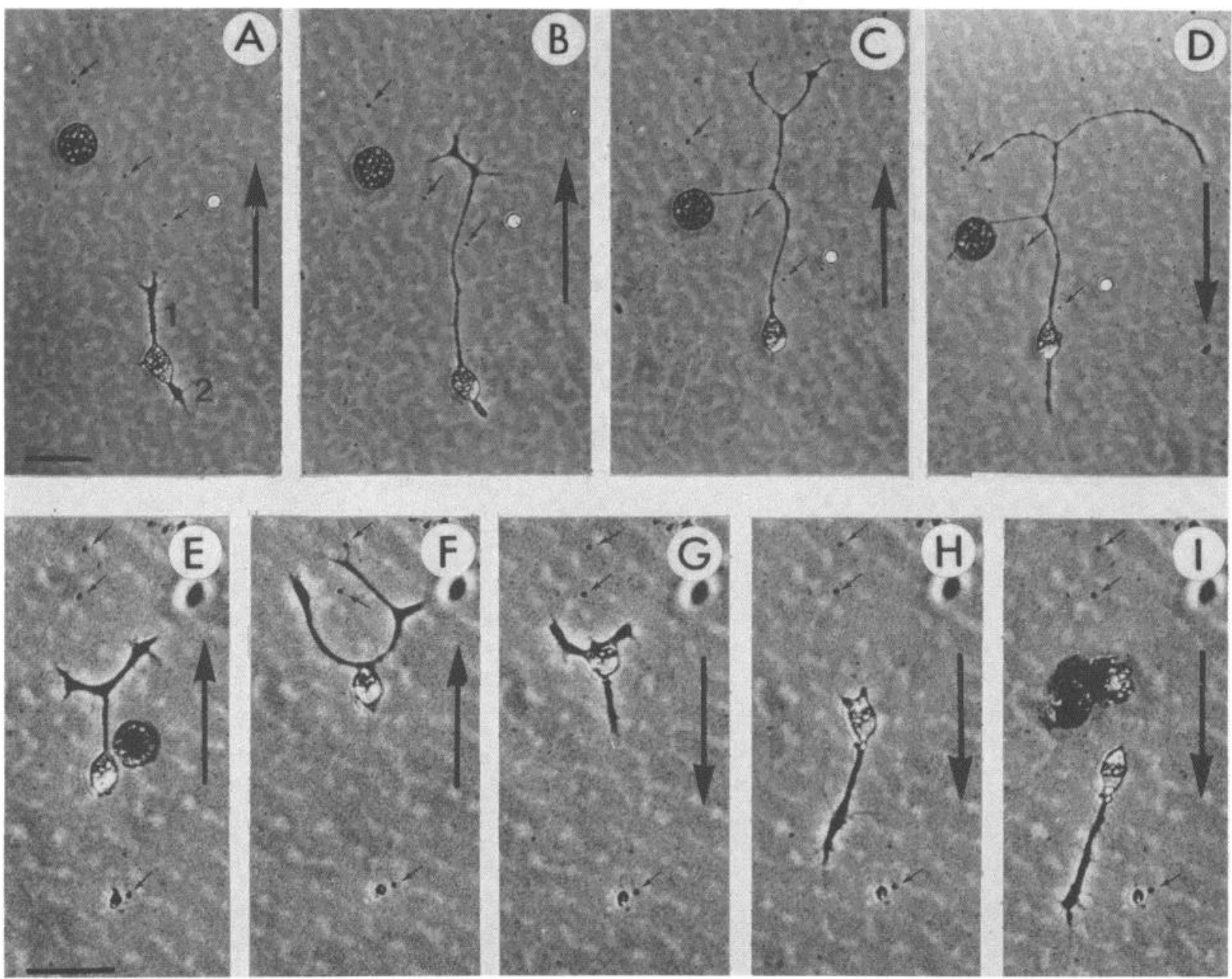

Figure 4. Asymmetric neurite growth and initiation of neurite outgrowth in the presence of an electric field (5 V/cm). $A$, A bipolar neuron (labeled 1 and 2) at the onset of the experiment. $B$, After $2 \mathrm{hr}$ exposure to the electric field. Neurite 1 (facing the cathode) grew substantially, while neurite 2 retracted. $C$, After $4 \mathrm{hr}$ exposure to the field. Neurite 1 grew further and branched, and neurite 2 disappeared. The polarity of the field was reversed immediately after the photograph shown in $C$ was taken. $D, 2$ $\mathrm{hr}$ after the field was reversed, the branched neurite 1 bent toward the new cathode and neurite 2 reappeared on the new cathodal side of the cell. $E$, A monopolar neuron at the onset of the experiment. $F$, After 2 hr exposure to the field. Further growth of the neurite, which was accompanied by the migration of the cell body toward the cathode. The field then was reversed. $G, 2$ hr after the field reversal, new neurite outgrowth appeared on the new cathodal pole of the cell, and the original neurites retracted extensively. $H, 4 \mathrm{hr}$ after the field reversal, the new neurite grew substantially, while the original neurite further retracted. $I, 6 \mathrm{hr}$ after the field reversal, there is further growth of the new neurite and a complete disappearance of the original neurite. Note that, in these two cells, substantial migration of the cell bodies was observed. The direction of cell migration was mostly toward the cathode and the cell bodies appeared to be "dragged" along by the growing neurites. Thick arrows represent the direction of the field, and thin arrows point to the fixed markers in the culture substratum. Bar, $50 \mu \mathrm{m}$.

surface of Xenopus neurons, like most cells, appears to have a net negative charge, since free floating neurons were seen to migrate toward the anode in manner consistent with the notion of cell electrophoresis. Thus, the imposition of an electric field parallel to the surface of cells which had adhered to the substratum should produce a flow of positive counter-ions toward the cathodal side of the cells, resulting in a steady medium flow over the cell surface. By observing the field-induced movements of suspended latex beads (diameter $=0.794 \pm$ $0.004 \mu \mathrm{m}$, Sigma) near the cell surface, we estimated that the electro-osmotic medium flow near the cell surface was no greater than $6 \mu \mathrm{m} / \mathrm{sec}$ for a field of $10 \mathrm{~V} / \mathrm{cm}$. To test whether the medium flow could cause neurite orientation, we passed a slow constant flow of the fresh culture medium through the culture chamber, using a peristaltic pump (LKB). Cultures of Xenopus neurons were exposed to a perfusion flow of 100 and $5 \mu \mathrm{m} / \mathrm{sec}$ for $6 \mathrm{hr}$ in the absence of the field. The neurite growth under these conditions was found to be symmetric (NGA = 0.00 , NISA $=0.03$; and NGA $=-0.04$, NISA $=0.04$, Table I). When a 100 and $20 \mu \mathrm{m} / \mathrm{sec}$ perfusion was applied to separate cultures in the same or opposite direction as the electric field, we found that the asymmetry in neurite growth was not significantly different from that observed in cultures exposed to the same electric field alone (Table I). Finally, we constructed a culture chamber in which the flow of fresh medium was introduced at a speed of $10 \mu \mathrm{m} / \mathrm{sec}$ to the culture in a direction perpendicular to the field axis at the middle portion of the culture chamber. In four separate experiments, oriented growth was observed for cells in the 


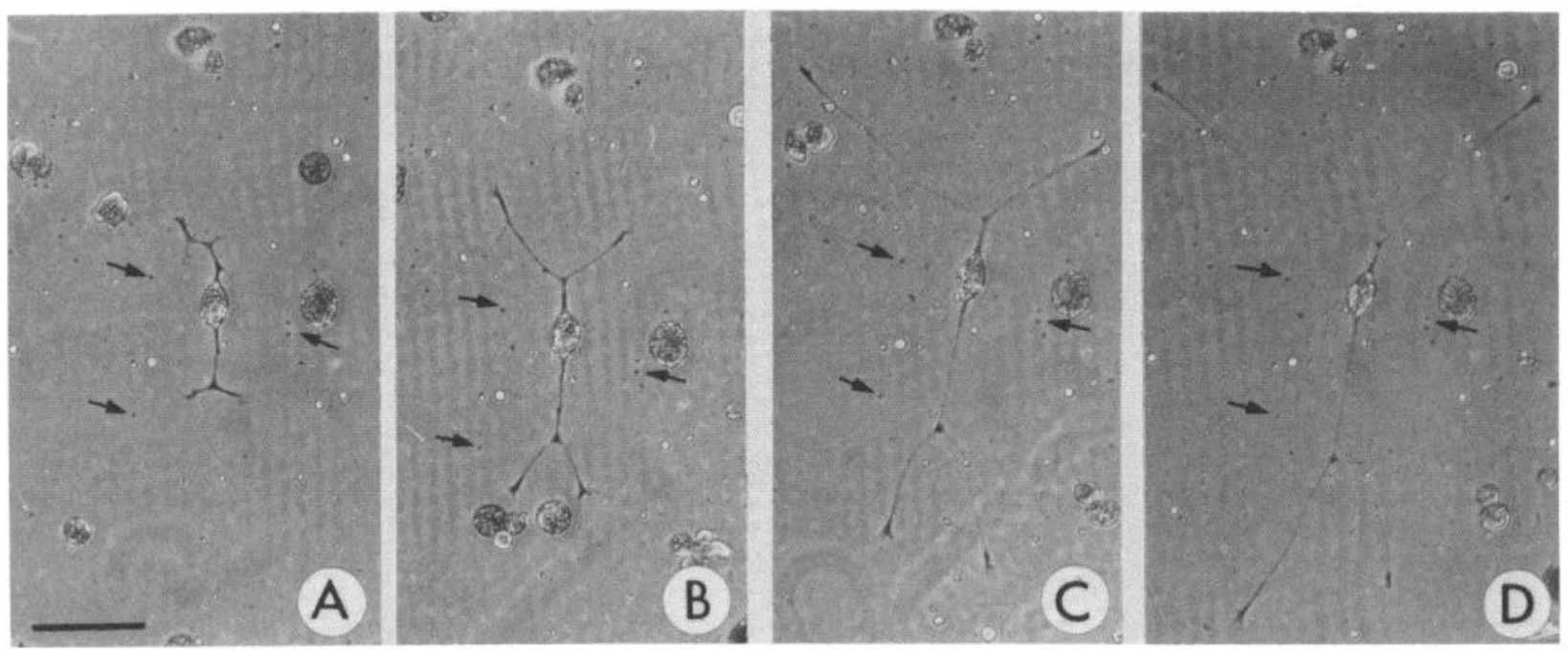

Figure 5. Neurite growth in a control culture (in the absence of the field). $A$, A bipolar neuron after a 5 -hr culture. $B$ to $D$, The same cell after 6,7 , and $8 \mathrm{hr}$ in culture, respectively. Note that the growth of two neurites were roughly symmetric. The arrows refer to fixed markers in the culture substratum. Bar, $50 \mu \mathrm{m}$.

middle of the chamber where they were exposed to both the medium flow and electric field for $6 \mathrm{hr}$ (Table I). These experiments suggest that gross medium flow cannot account for the observed orientation of neurite growth. Moreover, the use of constant perfusion of fresh culture medium through the culture chamber in the presence of the electric field also excluded the possibility that the neurite orientation was caused by a gradient of extracellular substances (chemical factors or ions) produced by the steady electric field. It should be noted that, even in the absence of imposed medium flow, a gradient of diffusible substances in the culture medium was unlikely to be established across the culture chamber by the electric field, since the two end pools of the chamber and the agar bridges contained enough culture medium to be considered as an infinite source of chemicals or ions over the duration of these experiments.

Threshold field for neurite orientation. Cultures of embryonic Xenopus neurons were treated with fields of various strength and duration. Both neurite growth asymmetry and neurite initiation site asymmetry increased as the duration of a particular field was increased. For a period of $24 \mathrm{hr}$, the smallest field that produced a neurite growth asymmetry (NGA $>0.10$ ) and asymmetry in the neurite initiation site (NISA $>0.10$ ) was 0.5 and $0.1 \mathrm{~V} / \mathrm{cm}$, respectively, which corresponds to a potential difference of 5 and $1 \mathrm{mV}$ across a distance of $100 \mu \mathrm{m}$ (a characteristic neurite length). By counting the number of cells showing asymmetric or curving neurite growth after exposure to an electric field for 16 to $20 \mathrm{hr}$, Hinkle et al. (1981) concluded that the threshold field for producing orienting effects was $0.07 \mathrm{~V} / \mathrm{cm}$, a value lower than that reported here. The discrepancy of results could simply be due to the difference in methods used for assaying growth asymmetry in the two studies.

Pharmacological agents and changes in ionic environment. The electric field will produce an asymmetric alteration of membrane potential across the neuron. For a field of $10 \mathrm{~V} / \mathrm{cm}$ and an average dimension for the cell body or growth cone of $10 \mu \mathrm{m}$, the cathodal side will be constantly depolarized by about $5 \mathrm{mV}$, while the anodal side will be hyperpolarized by $5 \mathrm{mV}$ (Poo, 1981). This asymmetric membrane potential may bias the ionic conductances of voltage-dependent sodium and calcium channels known to be present in these Xenopus neurons (Spitzer, 1979). It is conceivable that the observed neurite orientation in the electric field may be due to the asymmetric ionic conductance on either side of the neurite or the cell body. Sodium channels appear not to be involved, since tetrodotoxin (TTX), at a concentration that fully blocks sodium channels, was ineffective in preventing asymmetric neurite growth in the electric field (see Table II). Calcium channel blockers, such as cobalt and D600, used at their effective channel-blocking concentrations were cytotoxic to these embryonic neurons, resulting in the complete absence of neurites. The involvement of calcium channels in the observed neurite orientation thus remains a possibility.

Schubert et al. (1978) have suggested that calcium ions and cyclic adenosine monophosphate (cAMP) may regulate the neurite growth in pheochromocytoma (PC12) cell lines. We tested whether the asymmetric neurite growth produced in the electric fields could be the result of a corresponding intracellular gradient of $\mathrm{Ca}^{2+}$ or cAMP in these embryonic neurons created by the applied field. The cultures were incubated in medium containing 10 $\mathrm{mM} \mathrm{Ca}{ }^{2+}$, with and without calcium ionophore A23187 $(0.02 \mu \mathrm{g} / \mathrm{ml})$, or in medium containing $10 \mathrm{~mm}$ dibutyryl cAMP (dBcAMP). The high influx of $\mathrm{Ca}^{2+}$ through the $\mathrm{Ca}^{2+}$ ionophore into the neuron would presumably swamp out any intracellular $\mathrm{Ca}^{2+}$ gradient. Similarly, an intracellular cAMP gradient might be nullified by the entry of the membrane-permeable dibutyryl cAMP into the neuron. However, none of these experimental conditions prevented the asymmetric neurite growth and the asymmetric initiation of neurites in an electric field (Table II).

Cathodal accumulation of the surface Con $A$ receptors. Steady extracellular electric fields are known to cause migration of cell membrane components in the 
TABLE II

Neuronal response to the field in different pharmacological and ionic environments

\begin{tabular}{|c|c|c|c|c|c|c|c|c|}
\hline $\begin{array}{l}\text { Field Strength } \\
\text { and Duration } \\
\text { (hr) }\end{array}$ & Chemical Treatment & $\begin{array}{c}\text { Total } \\
\text { Neurons } \\
\text { Measured }\end{array}$ & $\begin{array}{c}\text { Total } \\
\text { Neurites } \\
\text { Measured }\end{array}$ & $\begin{array}{c}\text { Average Length } \\
\text { Projected toward } \\
\text { Cathode }^{a}\end{array}$ & $\begin{array}{l}\text { Average Length } \\
\text { Projected toward } \\
\text { Anode }^{a}\end{array}$ & $\begin{array}{l}\text { Average Neurite } \\
\text { Length }^{\alpha}\end{array}$ & $\begin{array}{c}\text { Asymmetry Index } \\
\text { for Neurite } \\
\text { Growth }^{b}\end{array}$ & $\begin{array}{l}\text { Asymmetry Index } \\
\text { for Neurite } \\
\text { Initiation Sites }^{b}\end{array}$ \\
\hline$V / \mathrm{cm}$ & & & & & $\mu m$ & & & \\
\hline $0.0(6)$ & TTX $(10 \mu \mathrm{M})$ & $11(1)^{r}$ & 17 & $40.7 \pm 8.0$ & $38.4 \pm 6.9$ & $75.8 \pm 16.8$ & $0.03(-)$ & $-0.10(-)$ \\
\hline $2.5(6)$ & TTX $(10 \mu \mathbf{M})$ & $82(2)$ & 140 & $64.8 \pm 5.0$ & $43.3 \pm 4.4$ & $89.5 \pm 8.0$ & $0.20(+)$ & $0.10(-)$ \\
\hline $0.0(6)$ & $10 \mathrm{mM} \mathrm{Ca}^{2+}$ & $174(3)$ & 328 & $52.1 \pm 2.8$ & $55.0 \pm 3.3$ & $79.7 \pm 6.1$ & $-0.03(-)$ & $0.05(-)$ \\
\hline $2.5(6)$ & $10 \mathrm{mM} \mathrm{Ca}^{2+}$ & $208(3)$ & 385 & $61.1 \pm 4.5$ & $39.6 \pm 3.3$ & $78.2 \pm 8.6$ & $0.22(+)$ & $0.13(t)$ \\
\hline $0.0(6)$ & $\begin{array}{c}10 \mathrm{~mm} \mathrm{Ca}{ }^{2+}, 0.02 \\
\mu \mathrm{g} / \mathrm{ml} \mathrm{Ca}^{2+} \\
\text { ionophore }\end{array}$ & $50(3)$ & 104 & $70.5 \pm 6.5$ & $63.3 \pm 6.2$ & $101.3 \pm 13.6$ & $0.05(-)$ & $-0.03(-)$ \\
\hline $2.5(6)$ & $\begin{array}{c}10 \mathrm{mM} \mathrm{Ca}^{2+}, 0.02 \\
\mu \mathrm{g} / \mathrm{ml} \mathrm{Ca}^{2+} \\
\text { ionophore }\end{array}$ & $143(3)$ & 282 & $82.3 \pm 4.2$ & $46.4 \pm 3.3$ & $94.8 \pm 7.4$ & $0.28(+)$ & $0.11(+)$ \\
\hline $0.0(12)$ & None & $58(3)$ & 81 & $67.1 \pm 6.9$ & $62.1 \pm 7.2$ & $113.8 \pm 14.8$ & $0.05(-)$ & $0.05(-)$ \\
\hline $0.0(12)$ & $10 \mathrm{~mm} \mathrm{dBcAMP}$ & $74(2)$ & 117 & $46.6 \pm 4.4$ & $38.6 \pm 3.3$ & $69.5 \pm 6.1$ & $0.09(-)$ & $0.03(-)$ \\
\hline $2.5(12)$ & None & $111(2)$ & 191 & $88.1 \pm 5.9$ & $56.4 \pm 3.8$ & $127.0 \pm 10.5$ & $0.22(+)$ & $0.13(t)$ \\
\hline $2.5(12)$ & $10 \mathrm{mM} \mathrm{dBcAMP}$ & $191(3)$ & 299 & $88.5 \pm 5.0$ & $46.1 \pm 4.2$ & $122.9 \pm 9.2$ & $0.32(+)$ & $0.19(+)$ \\
\hline $10(12)$ & None & $170(3)$ & 256 & $96.2 \pm 3.9$ & $41.4 \pm 3.4$ & $131.8 \pm 7.8$ & $0.40(+)$ & $0.26(+)$ \\
\hline $10(12)$ & $10 \mathrm{~mm} \mathrm{dBcAMP}$ & $161(2)$ & 231 & $101.3 \pm 4.7$ & $45.7 \pm 5.0$ & $133.0 \pm 8.6$ & $0.38(+)$ & $0.32(+)$ \\
\hline
\end{tabular}

"Values represent the mean $\pm \mathrm{SEM}$.

"Asymmetry indices were defined under "Materials and Methods." Indices $\leq 0.10$ were chosen to represent insignificant asymmetry ( - ), and indices $>0.10$ indicate significant asymmetry $(+)$.

"Number of cultures measured.

plane of cell membrane, a phenomenon termed "in situ electrophoresis" (Poo, 1981). It has been shown that, in the Xenopus embryonic culture, cell surface concanavalin $\mathrm{A}$ (Con $\mathrm{A})$ receptors can be induced to accumulate on the cathodal side of the myotomal cells by exposure of the cells to an electric field of 1 to $10 \mathrm{~V} / \mathrm{cm}$ (Poo et al., 1978,1979 ). Since electric fields of this magnitude were found in the present study to orient the neurite growth in the embryonic Xenopus culture, it was natural to inquire whether the field-induced migration of neuronal membrane components is involved in the observed orientation effect on neurite growth. We thus performed a series of experiments to examine the effect of the electric field on the distribution of the Con A receptors in the membrane of Xenopus neurons.

Embryonic Xenopus neuronal cultures were exposed to an electric field of $5 \mathrm{~V} / \mathrm{cm}$ in the same culture chamber as described under "Materials and Methods." Upon termination of the field, the cells were incubated immediately in saline containing $70 \mu \mathrm{g} / \mathrm{ml}$ of Con A conjugated with tetramethylrhodamine isothiocyanate (R-Con A) (Vector Laboratories) for $10 \mathrm{~min}$ at $4^{\circ} \mathrm{C}$. The distribution of R-Con A staining on the cell bodies then was measured by a microfluorimeter (Poo et al., 1979), and a normalized difference between the fluorescence intensity on the cathode and anode faces of the cell body was computed. Since Con A receptors could migrate from the cell body into the neurite or vice versa when the neurite is initiated directly toward the cathode or anode pole of the field, we chose to examine only those cells which initiated neurites relatively perpendicular $\left(60^{\circ}\right.$ to $\left.120^{\circ}\right)$ to the direction of the applied field in order to examine the field-induced migration on the cell bodies. Results showed that, after exposure to a field of $5 \mathrm{~V} / \mathrm{cm}$ for either $6 \mathrm{hr}$ or $30 \mathrm{~min}, \mathrm{R}$ Con A staining was greater on the cathode than the anode side of the cell bodies, suggesting an accumulation of the Con A receptors to the cathodal side of the cells (Table III and Fig. 6). When the cells were labeled with R-Con A $30 \mathrm{~min}$ after the termination of the field, there was no significant asymmetry in Con A receptor distribution (Table III), consistent with the notion that the

TABLE III

Migration of Con A receptors induced by the electric field

\begin{tabular}{|c|c|}
\hline Experimental Conditions $^{a}$ & $\begin{array}{c}\text { Average Asymmetry Index for Con A } \\
\text { Receptor Distribution }{ }^{b}\end{array}$ \\
\hline Control & $00 \pm 0$ \\
\hline $\begin{array}{l}\text { Cells labeled immediately after } \\
\text { removal of the field }(5 \mathrm{~V} / \mathrm{cm}, 6 \\
\text { hr) }\end{array}$ & \\
\hline $\begin{array}{l}\text { Cells labeled } 30 \mathrm{~min} \text { after re- } \\
\text { moval of the field }(5 \mathrm{~V} / \mathrm{cm}, 6 \\
\mathrm{hr})\end{array}$ & $0.07 \pm 0.06(N=34,4$ \\
\hline $\begin{array}{l}\text { Cells labeled immediately after } \\
\text { removal of the field }(5 \mathrm{~V} / \mathrm{cm} \text {, } \\
30 \mathrm{~min})\end{array}$ & $0.18 \pm 0.08(N=42,5$ cultures $)$ \\
\hline $\begin{array}{l}\text { Cells labeled before applying the } \\
\text { field ( } 5 \mathrm{~V} / \mathrm{cm}, 30 \mathrm{~min})\end{array}$ & $0.00 \pm 0.07(N=55,5$ cultures $)$ \\
\hline
\end{tabular}

a The cells were labeled with R-Con A $(70 \mu \mathrm{g} / \mathrm{ml})$ for $10 \mathrm{~min}$ at $4^{\circ} \mathrm{C}$ either before or after the exposure to the field and were examined immediately after labeling.

${ }^{b}$ Asymmelry indices were determined from microfluorimetric measurements of fluorescence intensity on the cathodal $\left(I_{c}\right)$ and anodal $\left(I_{a}\right)$ sides of the cell bodies of R-Con A by a microfluorimeter (PM1, Carl Zeiss, Inc., New York) with a $4-\mu \mathrm{m}$ aperture. The asymmetry index was defined as $\left(I_{c}-I_{a}\right) /\left(I_{c}+I_{a}\right)$. The background fluorescence from the adjacent cell-free region was subtracted from the readings made wilh the cells. Positive values indicate accumulation toward the cathode. $N$ refers to the total number of cells randomly sampled from separate cultures. The average asymmetry index is represented by the average value $\pm 95 \%$ confidence limits. 

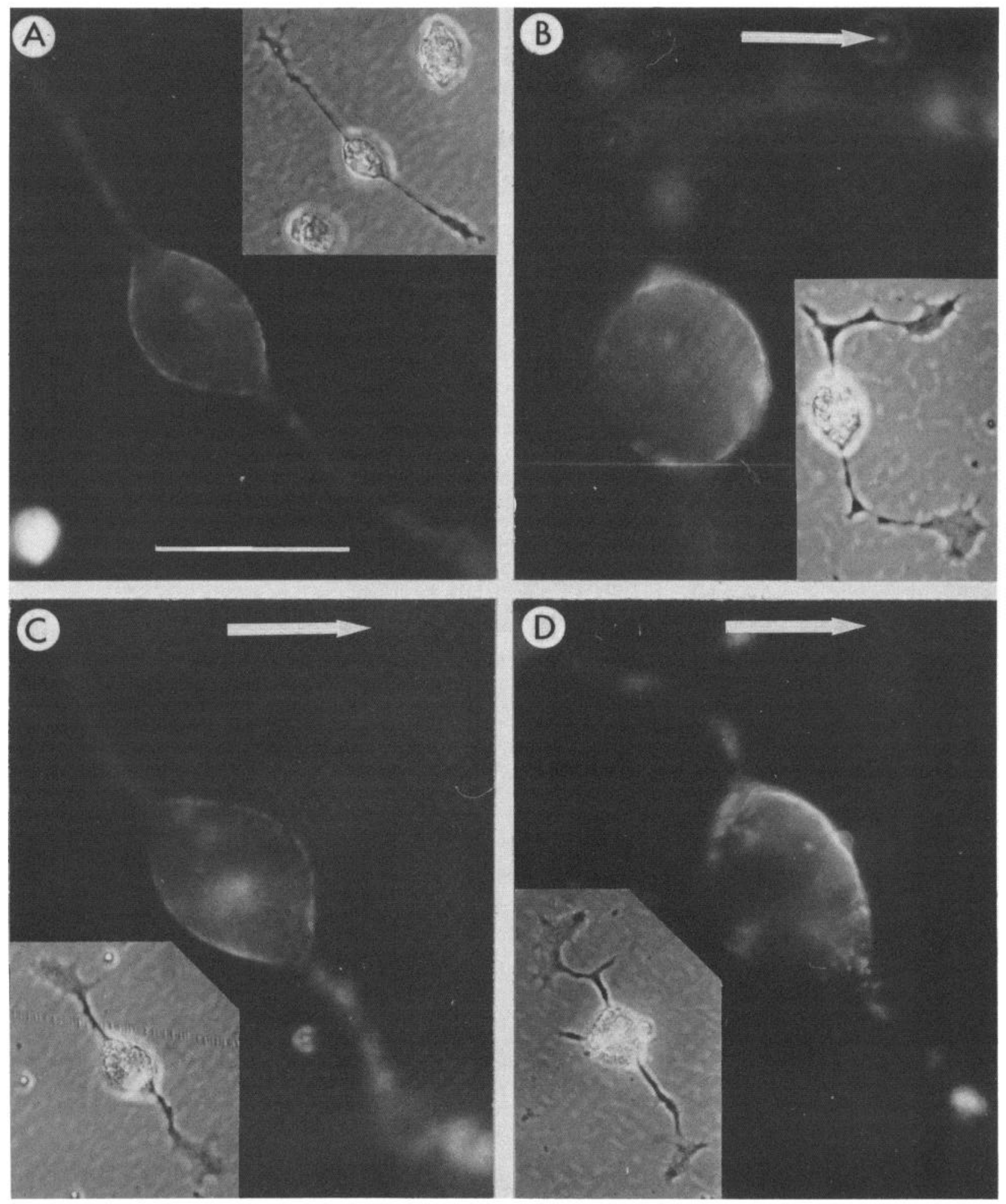

Figure 6. Migration of surface Con A receptors on the neuronal cell bodies induced by electric fields. Cells were labeled with R-Con A either before or after the exposure to an electric field. Photomicrographs were taken under epifluorescence and phase contrast optics for representative cells from the cultures. $A$, Cell from a $6-\mathrm{hr}$ control culture labeled immediately before examination. $B$, Cell from a 6 -hr culture that has been exposed to a field of $5 \mathrm{~V} / \mathrm{cm}$ for 6 hr. Labeling with R-Con A was carried out immediately after removal of the field. $C$, Cell from a 6-hr culture labeled with R-Con A before the exposure to a field of $5 \mathrm{~V} /$ $\mathrm{cm}$ for $30 \mathrm{~min}$. $D$, Cell from a 6-hr culture labeled with R-Con A after the exposure to a field of $5 \mathrm{~V} / \mathrm{cm}$ for $30 \mathrm{~min}$. Arrows mark the direction of the field. Note, in $B$ and $D$, marked accumulation of Con A receptors on the cathodal side of the cell bodies was seen. In contrast, the distribution of the Con A receptor in the control culture $(A)$ and the culture prelabeled with R-Con A $(C)$ appeared to be random on the cell surface. Bar, $20 \mu \mathrm{m}$. 
accumulated receptors undergo rapid back diffusion (Poo et al., 1979). Binding of R-Con A to neurons prior to the application of an electric field blocked the field-induced accumulation of the Con A receptors (Table III), a result that agrees with the previous finding on the Xenopus myotomal cells (Poo et al., 1979). This binding of R-Con $\mathrm{A}$, tetrameric ligand, presumably cross-linked and immobilized the Con A receptors on the cell surface. Taken together, the results indicate that a field of $5 \mathrm{~V} / \mathrm{cm}$ can cause significant migration of the pre-existing Con $\mathrm{A}$ receptors in the neuronal membrane toward the cathodal side of the cell.

Redistribution of the Con A receptors also may have occurred along the neurites. We found that, after exposure to the electric field, growth cones facing the cathode showed more numerous clusters of stain and higher intensity of the smoothly distributed stain than those facing the anode (Fig. 7). Since we did not carry out a quantitative assay of the number of filopodia or the surface area of the growth cone membrane facing the opposite poles of the field, it is unclear at present whether such asymmetric staining merely represents the difference in membrane morphology induced by the electric field or the accumulation of the Con A receptors at the tip of the neurite facing the cathode. The Con A receptors along the neurites facing the anode appeared to be depleted, since for the neurites of the same diameter and morphology (as observed in phase contrast optics), anode-facing neurites clearly showed much lower intensity of R-Con A stain than the cathode-facing ones (see Fig. 7).

Inhibition of field-induced growth orientation by Con $A$ binding. Cathodal accumulation of the Con A receptor was observed at field strengths that produced orientation of neurite growth toward the cathode. If field-induced Con A receptor accumulation is involved in the orientation response of the neurite growth, then incubation of the neurons with Con A during field application should prevent this response, since such treatment was shown to prevent the field-induced migration of the Con A receptors (see above).

Several cultures were incubated with culture medium containing 10 to $80 \mu \mathrm{g} / \mathrm{ml}$ of Con A and exposed to an electric field of $5 \mathrm{~V} / \mathrm{cm}$ for $6 \mathrm{hr}$. The average neurite length, neurite growth asymmetry, and neurite initiation site asymmetry were determined. The results are shown in Figure 8. The presence of Con A resulted in a slight reduction in the average neurite length. However, a striking absence of asymmetry in neurite growth and initiation site was observed for cells incubated with Con $\mathrm{A}$ at
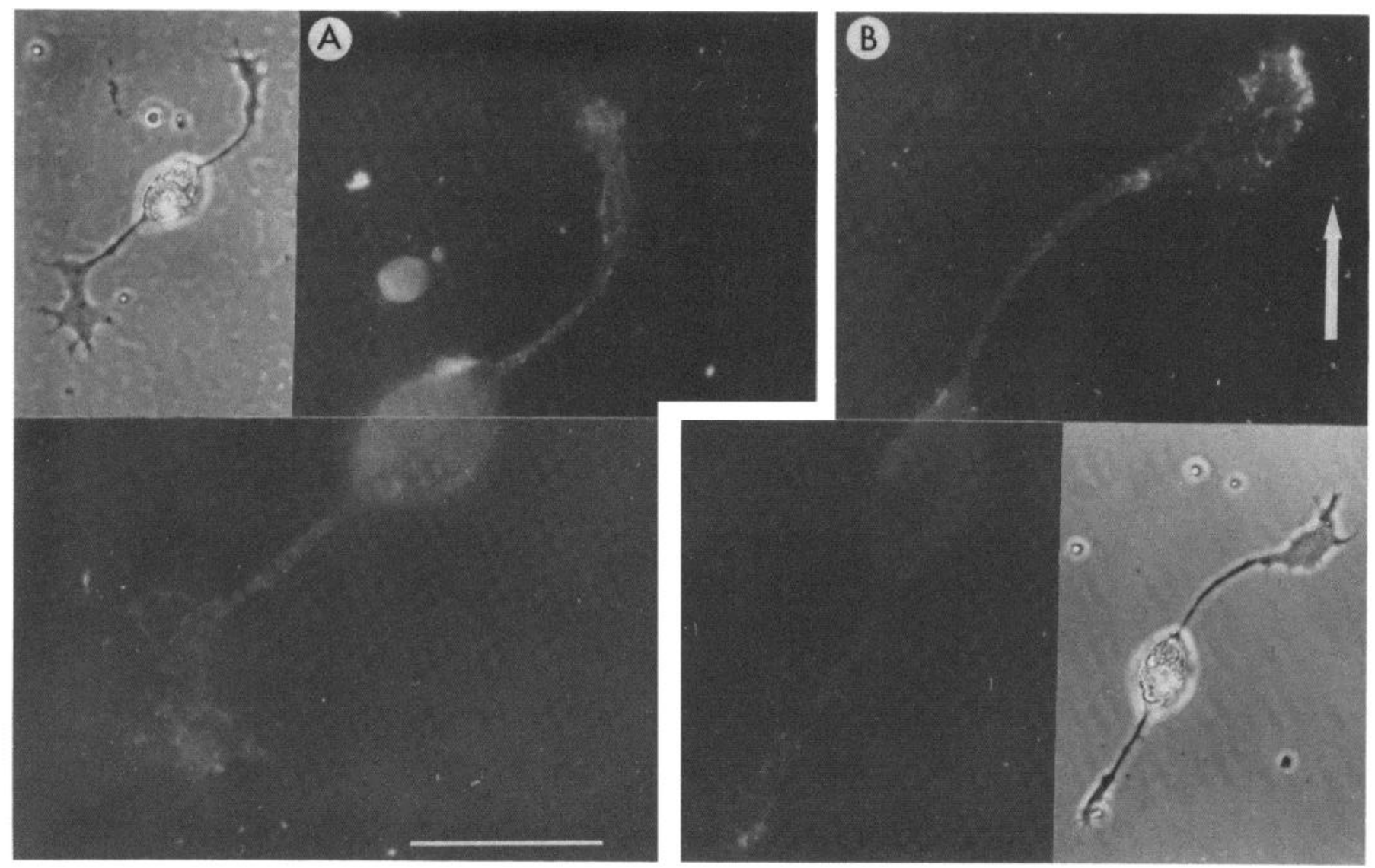

Figure 7. Distribution of the Con A receptors along neurites. A, A bipolar neuron in a 6-hr control culture labeled with R-Con A immediately before the observation. $B$, A bipolar neuron in a $6-\mathrm{hr}$ culture that has been exposed to a field of $5 \mathrm{~V} / \mathrm{cm}$ for $6 \mathrm{hr}$. The cell was labeled with R-Con A after removal of the field and examined immediately after the labeling. The arrow indicates the direction of the field. Note the accumulation of R-Con A staining at the neurite tip and along the neurite facing the cathode. Bar, $20 \mu \mathrm{m}$. 


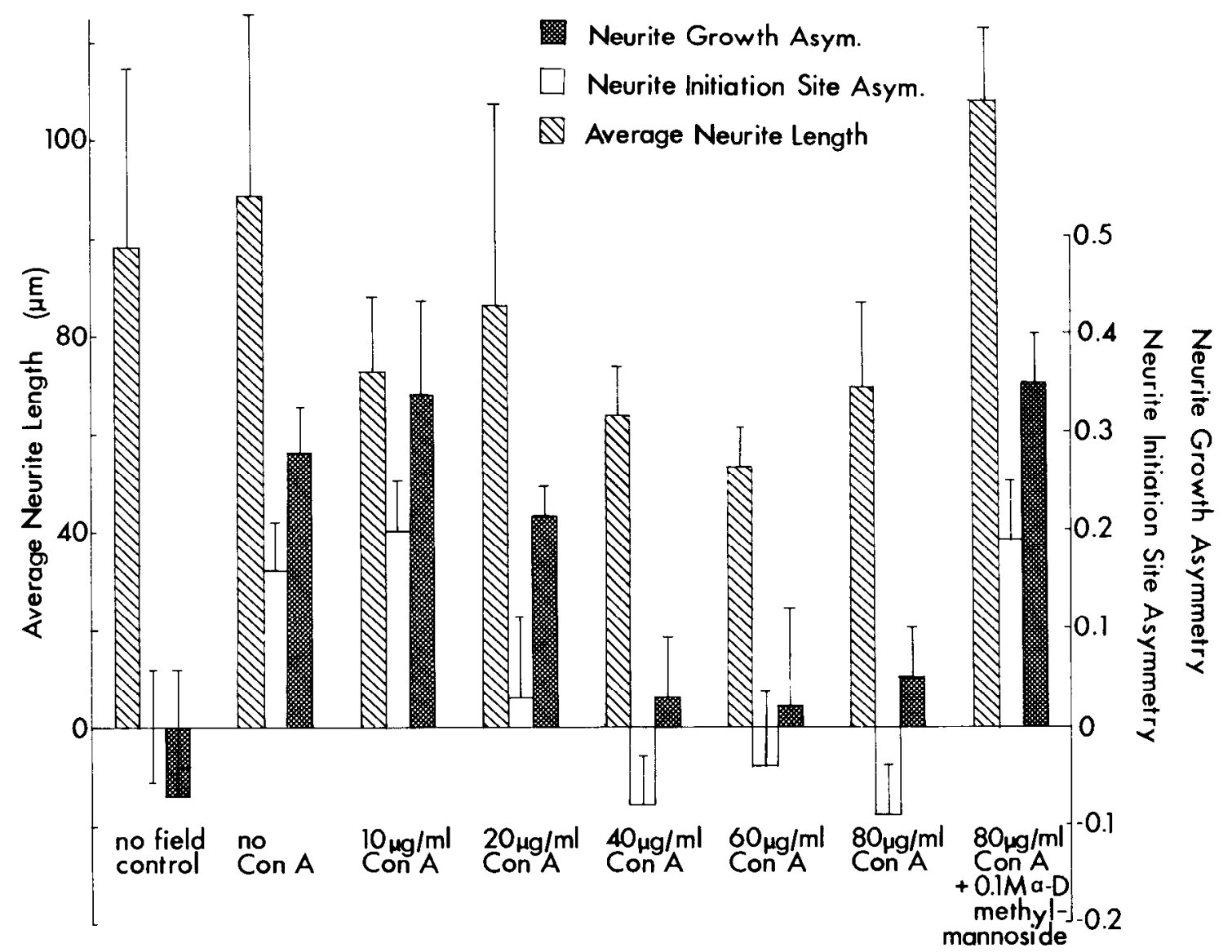

Figure 8. Electric field effects on neurite growth in the presence of Con A. A field of $5 \mathrm{~V} / \mathrm{cm}$ was applied to a series of cultures for $6 \mathrm{hr}$ in the presence of various concentrations of Con A and the neurite growth responses were examined for the average neurite length (in micrometers), the asymmetry in neurite growth, and asymmetry in the neurite initiation sites (see "Materials and Methods"). The average neurite length was slightly reduced by the presence of Con A, while the field-induced asymmetry in neurite growth and initiation site was completely abolished at $80 \mu \mathrm{g} / \mathrm{ml}$ of Con $\mathrm{A}$. The Con A effect was specific, since after incubation in Con A, together with Con A-specific sugar, $\alpha$-D-methylmannoside, a high neurite growth asymmetry was observed. For asymmetry in neurite growth and neurite initiation site, each data point represents an average of indices measured from three to five separate cultures. For average neurite length, each data point represents the average of all neurites from three to five separate cultures. The total number of neurites per culture ranged between 17 and 60 . The error bars represent the SEM of a $95 \%$ confidence level.

levels above $20 \mu \mathrm{g} / \mathrm{ml}$. This inhibition effect is due to specific binding of the Con A to its receptor, since incubation of the culture containing a high concentration of Con A $(80 \mu \mathrm{g} / \mathrm{ml})$ and $\alpha$-D-methylmannoside $(0.1 \mathrm{M})$, a Con A-specific sugar, resulted in high average growth and strong growth orientation in the presence of the field.

\section{Discussion}

The present study has demonstrated quantitatively three effects of steady extracellular electric fields on the neurite growth from individual Xenopus embryonic neurons in culture: (1) neurite growth was accelerated toward the cathode but reduced toward the anode, and the growing tips were prompted to bend toward the cathode, causing the neurites to curve toward the cathode; (2) more neurites appeared to be initiated on the cathodal side of the soma; and (3) the number of neurite-bearing neurons and the average length of neurite in the culture were increased.

Our finding of the asymmetry in neurite growth agrees qualitatively with previous studies using explanted nervous tissue (Marsh and Beams, 1946; Sisken and Smith, 1975; Jaffe and Poo, 1979). Recently, Hinkle et al. (1981) have reported a similar study on the electric field effects on cultured Xenopus neurons. The approach used by Hinkle et al. was simply to count the number of cells in the culture showing preferential growth or bending toward the cathode; no actual measurement of neurite length was carried out. Their results on the preferential growth, curving response of the neurite, and stimulation of neurite outgrowth agree qualitatively with those reported here. However, they failed to observe the polari- 
zation in neurite initiation sites on the cell bodies after treatment of the cell with a field for 2 to $24 \mathrm{hr}$. Although the apparent polarization observed in our study could have resulted in part from the prevention of neurite initiation on the anodal side of the cell or from the retraction of anode-facing neurites, time-lapse studies (Fig. 4, $E$ to $I$ ) showed that the polarity of an "apparent" monopolar neuron can be reversed by the field, suggesting that de novo creation of a neurite initiation site on the cell body also may occur in our culture. Further studies using a homogeneous population of neurons with a well characterized pattern of neurite outgrowth is needed to clarify this polarization effect.

In a paper summarizing various environmental factors that determine the course of growing nerve fibers in vitro, Weiss (1934) concluded that the primary cause of the earlier observation on the orientation of the nerve growth by an electric field (Ingvar, 1920) was the mechanical guidance provided by oriented micelles in the culture medium, which resulted from the application of the field. However, it is difficult to envision how such mechanical guidance could simultaneously enhance growth toward the cathode and inhibit growth toward the anode. Results from our experiments with constant perfusion of the culture, especially that of the perfusion perpendicular to the field axis, also argue against the possibility that medium flow serves as a mechanical force in producing neurite orientation. Finally, the finding that the specific binding of Con A molecules to the neuronal membrane abolished the field-induced neurite orientation makes it extremely unlikely that the orientation resulted from any secondary mechanical factor created by the electric field in the growth environment of the neurite.

The oriented neurite growth that we observed could not have resulted from field-induced gradients of ions and molecules in the culture medium or of factors released by the cells, since constant perfusion of fresh medium in the same, the opposite, and perpendicular direction with respect to the field axis all failed to affect the oriented growth induced by the field. Thus, we are led to the conclusion that the field must act directly on these neurons to produce an oriented neurite growth.

There are three possible ways that an extracellular electric field could act directly on a neuron. First, the electric field may act intracellularly by producing a cytoplasmic potential drop that could cause electrophoretic redistribution of cytoplasmic materials involved in neurite growth. Second, it may alter the cell's membrane potential asymmetrically and therefore preferentially activate growth-controlling transport processes across the plasma membrane. Third, it also may act along the plasma membrane, causing an electrophoretic accumulation of the surface molecules responsible for neurite growth control or cell-substratum adhesion. The plausibility of these field actions in accounting for our observed neurite orientation is discussed below.

Since the resistivity of the cytoplasm is much lower than that of the plasma membrane, the intracellular potential drop is on the order of $10^{-4}$ of the extracellular potential applied across the cell (Poo, 1981). The fields $(0.10$ to $10 \mathrm{~V} / \mathrm{cm})$ used in our experiments thus produced an intracellular potential difference of about 0.01 to $1 \mu \mathrm{V}$ across a cell or a growth cone $10 \mu \mathrm{m}$ in width. Although these fields are small, they could produce gradients of cytoplasmic material over a prolonged duration. For example, to move a charged component (macromolccule or vesicle) of usual electrophoretic mobility $(1 \mu \mathrm{m} / \mathrm{sec} / \mathrm{V} /$ cm) across a $10-\mu \mathrm{m}$ distance requires $10^{4}$ to $10^{6} \mathrm{sec}(3 \mathrm{hr}$ to $10 \mathrm{~d}$ ). Thus, it is conceivable that, in our experiments, especially those using higher field strengths (e.g., 5 and $10 \mathrm{~V} / \mathrm{cm}$ ), significant intracellular migration of material could have occurred. A slight asymmetry in the distribution of cytoplasmic membrane precursors may, in fact, be enough to trigger a "bending" of the growth cone within $1 \mathrm{hr}$ (Figs. 3 and 4 ).

The action of the field across the membrane also should be seriously considered, since most of the potential drop produced by the applied field occurs across the plasma membrane. For a field strength of 1 to $10 \mathrm{~V} / \mathrm{cm}$ applicd across a cell body or growth cone $10 \mu \mathrm{m}$ wide, the membrane potential on the cathodal side of the cell will be depolarized by about 0.5 to $5 \mathrm{mV}$; that facing the anode will be hyperpolarized by the same amount (Poo, 1981). This steady asymmetric alteration of membrane potential could bias ionic conductances or enzyme activation (or both) involved in the regulation of neurite growth on the two sides of the cell. Specifically, we considered the possibility that a cytoplasmic gradient of $\mathrm{Ca}^{2+}$ or cAMP could be created by a preferential influx of $\mathrm{Ca}^{2+}$ or activation of adenylate cyclases on the cathodal (depolarized) side of the plasma membrane (Table II). We have attempted to swamp out these gradients by using high extracellular $\mathrm{Ca}^{2+}$ (in the presence of a calcium ionophore) or dBcAMP. The results suggest that the cytoplasmic gradient of $\mathrm{Ca}^{2+}$ or cAMP may not be involved in the field effects.

In addition to the above transmembrane action, the tangential (perimembrane) component of the extracellularly applied electric field could act along the plasma membrane of the cell. It has been shown to produce electrophoretic accumulation of surface glycoproteins in the isolated tissue culture cells (Poo, 1981). Since membrane glycoproteins are believed to play a crucial role in the cell's adhesion to other cells and to the substratum, a preferential cathodal accumulation of surface molecules involved in the neurite's adhesion to the culture substratum could be responsible for the orienting effect of the field. Alternatively, cathodal accumulation of membrane receptors for trophic substances (in the culture medium) could cause the observed asymmetric neurite growth. This possibility of lateral electrophoresis as an underlying mechanism also has been suggested by Jaffe and Poo (1979) and Hinkle et al. (1981). The present report represents the first demonstration that the electric field could cause migration of surface molecules of the neurons. Binding studies using fluorescently labeled Con A showed that the electric field similar to that used in orienting neurite growth produces an accumulation of the Con A receptors, presumably glycoproteins, on the cathodal surface of these neurons. This field-induced migration of surface Con A receptors may be related causally to the observed orientation effect of the field on neurite growth, since prevention of Con A receptor migration abolished the orientation effect.

In conclusion, we have carried out a quantitative study 
of the effects of electric fields on the orientation of neurite growth in a simple culture system of dissociated cells. We have demonstrated that these effects are due to direct action of the field on the neuron. Moreover, we have investigated various possible cellular mechanisms underlying these field effects and found a correlation between the field-induced migration of neuronal membrane Con A receptors and the growth orientation. Interestingly, applications of minute electric currents in vivo have been found to enhance greatly regeneration of injured nerves in amputated frog forelimb (Borgens et al., 1977) and in transected lamprey spinal cord (Borgens et al., 1981). The field strength produced within the tissue by the applied current in these in vivo studies is close to that used in our present study (see Borgens et al., 1981). The cellular mechanisms suggested by our result also may be responsible for the enhancement of nerve regeneration in vivo by small exogenous electric fields. However, whether endogenous electric fields within the tissue (Jaffe and Nuccitelli, 1977) play a role in orienting nerve growth in vivo and whether the orientation of nerve growth by chemical factors (Gundersen and Barrett, 1980) involves cellular mechanisms similar to that for the orientation by electric fields remains to be elucidated.

\section{References}

Borgens, R. B., J. W. Vanable, Jr., and L. F. Jaffe (1977) Bioelectricity and regeneration. 1. Initiation of frog limb regeneration by minute currents. J. Exp. Zool. 200: 403.

Borgens, R. B., D. E. Roederer, and M. J. Cohen (1981) Enhanced spinal cord regeneration in lamprey by applied electric fields. Science 213: 611-617.

Gundersen, R. W., and J. H. Barrett (1980) Characterization of the turning response of dorsal root neurites toward nerve growth factor. J. Cell Biol. 87: 546-554.

Hinkle, L., C. D. McCaig, and K. R. Robinson (1981) The direction of growth of differentiating neurons and myoblasts from frog embryos in an applied electric field. J. Physiol. (Lond.) 314: 121-135.
Ingvar, S. (1920) Reaction of cells to galvanic current in tissue culture. Proc. Soc. Exp. Biol. Med. 17: 198-199.

Jacobson, M. (1978) Developmental Neurobiology, Ed. 2, pp. 157-166, Plenum, New York.

Jaffe, L. F. (1958) Tropistic responses of zygotes of the fucaceae to polarized light. Exp. Cell Res. 15: 282-299.

Jaffe, L. F., and R. Nuccitelli (1977) Electrical controls of development. Annu. Rev. Biophys. Bioeng. 6: 445-476.

Jaffe, L. F., and M. -m. Poo (1979) Neurites grow faster towards the cathode than the anode in a steady field. J. Exp. Zool. 209: 115-128.

Jones, K. W., and T. R. Elsdale (1963) The culture of small aggregates of amphibian embryonic cells in vitro. J. Embryol. Exp. Morphol. 11: 135-154.

Marsh, G., and H. W. Beams (1946) In vitro control of growing chick nerve fibers by applied electric currents. J. Cell. Comp. Physiol. 27: 139-157.

Nieuwkoop, P. O., and J. Faber (1962) Normal Table of Xenopus laevis, North Holland Publishing Co., Amsterdam.

Patel, N. B., and M. -m. Poo (1979) Orientation of axonal growth of embryonic Xenopus neurons by small extracellular electric fields. Soc. Neurosci. Abstr. 5: 561.

Poo, M. -m. (1981) In situ electrophoresis of membrane components. Annu. Rev. Biophys. Bioeng. 10: 245-276.

Poo, M. -m., W. -j. H. Poo, and J. W. Lam (1978) Lateral electrophoresis and diffusion of concanavalin A receptors in the membrane of embryonic muscle cell. J. Cell Biol. 76: 483501.

Poo, M. -m., J. W. Lam, N. Orida, and A. W. Chao (1979) Electrophoresis and diffusion in the plane of the cell membrane. Biophys. J. 26: 1-22.

Schubert, D., M. La Carbieve, C. Whitloch, and W. Stallcup (1978) Alterations in the surface proteins of cells responsive to nerve growth factor. Nature 273: 718-723.

Sisken, B. F., and S. D. Smith (1975) The effect of minute direct electrical currents on cultured chick embryo trigeminal ganglia. J. Embryol. Exp. Morphol. 33: 29-41.

Spitzer, N. C. (1979) Ion channels in development. Annu. Rev. Neurosci. 2: 363-397.

Weiss, P. (1934) In vitro experiments on the factors determining the course of the outgrowing nerve fiber. J. Exp. Zool. 68: 393-448. 